\title{
Grand Challenges and Opportunities in Sensor Science and Technology
}

\author{
Dermot Diamond * \\ Insight Centre for Data Analytics, National Centre for Sensor Research, Dublin City University, Dublin, Ireland
}

Keywords: sensors, transducers, chemical sensors, biosensors, microfluidics, sensor data, sensing systems, societal impact

Sensors and sensing systems play a fundamental role in shaping how modern society functions. Sensors confer "smartness" to everyday objects, devices, and systems-things that we use and depend on every day. Sensors are the foundation of the so-called "Internet of Things," wherein objects and people become sensorised and linked through ubiquitous digital communications infrastructure. Parameters like temperature, light intensity/color/imaging, location, movement, proximity, can now be tracked over long time periods (years) and at huge scale, thanks to the availability of low-cost, highly-reliable sensors. Increasingly, these sensors function in an embedded mode, invisible to the user, but forming the basis of a multitude of Apps related to smart homes, smart cars, remote monitoring of weather and the environment, smart agriculture, supply chain logistics, food quality, and tracking personal health and condition. Integrating actuators with these monitoring systems opens the way to smart control-from local climate control in the home via switchable heating and air-conditioning systems, to self-driving cars, smart lawnmowers, remote surgery, and robotic assistants. Patterns of inter-dependency can now be investigated from very localized to global scale. For example, cause and effect can be explored across health and the environment, water quality and food supply, ocean acidity and atmospheric $\mathrm{CO}_{2}$, dynamics of disease spread and societal behavior, and so on. This link between very local and global will become a key topic for research, based on advances in physical transducer performance, particularly reliability in autonomous operation over extended use periods (years). Society is changing like never before, due to the merging of knowledge and ideas from multiple dimensions, particularly global-scale computing, high-speed digital communications with massive capacity, embedded sensing technologies, new rapid manufacturing and scale-up technologies, and advances in fundamental materials science.

At present, despite advances in terms of range of accessible target species, and improvements in selectivity and sensitivity, the same cannot be said for chemical sensing and biosensing, principally because of limited stability due to what could be called the biochemical sensor paradox (Byrne and Diamond, 2006), i.e.,

- In order to provide a predictable signal for a particular concentration of a target species in a sample, the sensor surface should not change over time;

Correspondence:

Dermot Diamona dermot.diamond@dcu.ie

Received: 22 May 2020

Accepted: 26 June 2020

Published: 05 August 2020

Citation:

Diamond D (2020) Grand Challenges

and Opportunities in Sensor Science

and Technology. Front. Sens. 1:2.

doi: 10.3389/fsens.2020.00002

But at the same time;

- In order to provide an analytically useful signal, the surface must selectively bind target molecules-it must change!

Consequently, biosensors and chemical sensors function on the basis of surfaces that must simultaneously responsive and passive.

Furthermore, these devices are typically exposed to complex sample media that contain many potential interfering species that compete with the target molecule for surface binding sites, as well as other sample components that coat or contaminate the sensor surface, for example, through biofouling in blood measurements or wastewater monitoring. 
Consequently, the dominant use model for many molecular measurements (and particularly biosensors) is single-shot measurements with disposable devices. In cases where continuous monitoring is possible (mainly chemical sensing), the use model requires regular servicing and calibration, which drives up the cost of ownership and severely limits our ability to implement large-scale deployments, and to provide simple devices capable of non-specialist operation at point-of-need. Calibration requires the incorporation of one or more standard solutions that must be diverted to contact the sensor surface in place of the sample, enabling aging effects to be quantified and corrected. In turn, this means that these sensing systems must incorporate a liquid handling system, including pumps and valves, that extend the service interval but simultaneously increase system complexity and cost.

The COVID-19 pandemic illustrates some of the challenges facing biosensing, such as the critical need for rapid diagnostics, and the long sample turn-around time associated with centralized specialist biochemical laboratory model compared to simple cloud-linked measurements at point-of-need (Udugama et al., 2020). Confirmation of infection begins with sampling by taking swabs which are then sent to remote, centralized labs for PCR-based testing. Issues with reagent availability, sample viability in storage, availability of trained staff to perform the measurements, and reporting of results (IT systems) a pinch point at any place along the measurement pathway stops the entire process leading to loss of confidence in the community when publicly announced targets are not met. In contrast, much simpler serological (antibody tests) allow accurate estimations of the level of community infection to be established. Making these low-cost, with widespread availability at local pharmacies, simple to use (non-specialist model similar to the pregnancy test), and the sensor measurement integrated with a mobile phone (e.g., phone camera detects outcome by imaging a clip-on test strip) enables personalized health data to become rapidly available via cloud based apps at a global scale. Specialist facilities operated by appropriately qualified and experienced staff will always be required to provide data validation via gold-standard measurements, and to perform complex measurements for which a simple biosensor does not currently exist. But as evidenced by the COVID-19 pandemic, there is a tremendous need, and therefore market opportunity, to move many personal health diagnostic tests away from specialist labs into the community. This is also true for other application domains, like environmental monitoring, food quality tracking, and ensuring pharmaceutical product stability. And while single-shot measurements with disposable sensors are ideal for confirmatory testing (yes/no outcomes), in many application scenarios, continuous monitoring over time is required. Therefore, the trend toward wearable patch type devices that continuously sample interstitial fluid through the skin, and provide real-time tracking of critical biomarkers via integrated electrochemical biosensors will continue to grow. This platform technology is replacing finger-prick sampling coupled with single use biosensors for diabetics to track their glucose levels, and optimize their insulin therapy. Clearly, underpinning these trends is the generic push toward "big data," cloudbased sensing and data, coupled with developments in Artificial Intelligence and Machine Learning that enable these huge data volumes to be explored, and inter-dependencies and patterns revealed at a scale unimaginable even a few years ago. Societal use and adoption of these new technologies must be safeguarded through close attention to issues like privacy, ethics, and trust, to ensure the tremendous power of these technologies is not abused (Robles et al., 2020).

From this brief overview, the breadth and depth of sensor related research and development, and the impact of sensor related technologies on society is truly breathtaking. But we are on the cusp of more profound impacts based on technologies and concepts that are now in their infancy, but will lead to dramatic changes in society in the coming years. Nanobots could well become a reality-nano-scaled entities that can be implanted in the body, or seeded into the environment, constantly scanning for signs of infection or pollution, responding to suspicious signals, dealing with low level situation locally, or escalating as required, to more powerful external resources, in effect, borrowing from the complex biofeedback mechanisms of the immune system, and applying them to augment health, maintain the environment, or create sophisticated behavior in devices like self-awareness and self-repair to extend functional lifetime, and only signaling for external intervention when necessary. We also need to delve into philosophical questions-what is a sensor? Is it a device, a material, a molecule? Or is it all of these and more? Can sensors be regarded as "alive," as they increasingly are conferred with characteristics normally associated with living things, such as programmed "intelligent" movement in response toward the source of a molecular plume? This coupled with triggered release of precursors, catalysts, and other molecular payloads with specific functions could provide the basis of awareness and self-maintenance, admittedly rather primitive initially, but constantly evolving toward more complex behavioral traits. Control of a local molecular environment could be implemented completed in the materials domain, in which materials sense and respond by releasing specific molecules without recourse to electronics is an exciting prospect.

Thought leaders in sensor science will be encouraged to present opinion on how advances in sensing technologies may impact society in the next $5,10,20$, or 50 years? Connected to this will be the impact of new fabrication technologies that could revolutionize sensor manufacturing, driving down unit production costs while also maintaining or improving product reliability. The pathway to market is also advancing, traditional barriers between the communities involved in research, innovation, company formation, product development and scale-up, and market knowledge are disappearing. Combined with a more agile and integrated funding environment from both public grant agencies and private investors, new sensing technologies and innovative services based on sensors can be supported through the at times difficult, but critical connection between a technology breakthrough and a market need. 
The suite of Frontiers in Sensors ${ }^{1}$ journals covers all of these topics-a timely offering that will make a major contribution to the entire range of sensor research-making fundamental knowledge emerging from the best laboratories readily available to the research community, and inviting the finest creative minds in sensor science to speculate on where the future

${ }^{1}$ https://www.frontiersin.org/journals/sensors

\section{REFERENCES}

Byrne, R., and Diamond, D. (2006). Chemo/bio-sensor networks. Nat. Mater. 5, 421-424. doi: 10.1038/nmat1661

Robles, T., Bordel, B., Alcarria, R., and Sánchez-de-Rivera, D. (2020). Enabling trustworthy personal data protection in eHealth and well-being services through privacy-by-design. Int. J. Distribut. Sensor Netw. 16:155014772091211. doi: $10.1177 / 1550147720912110$

Udugama, B., Kadhiresan, P., Kozlowski, H. N., Malekjahani, A., Osborne, M., Li, V. Y. C., et al. (2020). Diagnosing COVID-19: the disease and tools for detection. ACS Nano 14, 3822-3835. doi: 10.1021/acsnano.0c02624 impacts are likely to happen, and to identify key scientific and technological challenges that must be overcome on the journey to practical implementation.

\section{AUTHOR CONTRIBUTIONS}

The author confirms being the sole contributor of this work and has approved it for publication.

Conflict of Interest: The author declares that the research was conducted in the absence of any commercial or financial relationships that could be construed as a potential conflict of interest.

Copyright (C) 2020 Diamond. This is an open-access article distributed under the terms of the Creative Commons Attribution License (CC BY). The use, distribution or reproduction in other forums is permitted, provided the original author(s) and the copyright owner(s) are credited and that the original publication in this journal is cited, in accordance with accepted academic practice. No use, distribution or reproduction is permitted which does not comply with these terms. 